\title{
A rare case of extradural thoracic spinal chordoma with medullary compression and literature review
}

\author{
Amalia Ene ${ }^{1,2}$, Iulia Elena Petre ${ }^{1}$, Aurelia Nicoleta Dabu²,3 \\ ${ }^{1}$ Department of Neurology, Emergency University Hospital, Bucharest, Romania \\ 2"Carol Davila" University of Medicine and Pharmacy, Bucharest, Romania \\ ${ }^{3}$ Department of Neurosurgery, Emergency University Hospital, Bucharest, Romania
}

\begin{abstract}
Background. Chordomas are low-grade, slow-growing, but locally invasive and aggressive malignant tumors, arising from the remnants of the embryonic notochord. Given its origin, the distribution of chordomas is along the axial skeleton: $50 \%$ of cases in the sacral region, $35 \%$ skull base and $15 \%$ occur in the vertebral bodies of the spine, which most commonly involve cervical (particularly $\mathrm{C} 2$ ), followed by lumbar, and then the thoracic spine. Without a prompt and aggressive treatment the chances of metastasis are low, but the local recurrence rate is high (about $50 \%$ ) with a $40 \%$ chance of 10 -year survival.

Objective. Our purpose is to present a rare case of an extradural thoracic chordoma, the importance of differential diagnosis and the management of this case for a better outcome.

Case presentation. A 61 -year-old man presented with a 1-month history of thoracic back pain, followed by rapidly progressive paraparesis and paresthesia in both lower limbs, and 24 hour urinary retention. MRI of the thoracic spine revealed an contrast-enhancing lesion arising from T5 to T6 vertebral level, with pre and paravertebral extension and medullary compression. The diagnosis of chordoma was made by tissue biopsy and histopathological study. There are few reports of chordomas with this localization in English literature. After surgery, the patient showed slightly good clinical outcome in terms of improvement of muscle strength in lower limbs and back pain decrease in intensity of back pain.

Conclusions. Primary chordoma in the thoracic spine is extremely rare (less than $15 \%$ of the cases). This case highlights the importance of emergency surgery in the treatment of spinal tumors and the need to consider chordoma as a differential diagnosis for extradural spinal tumors. On the other hand the treatment is challenging because the current literature is lacking in evidence based guidelines due to low incidence.
\end{abstract}

Keywords: chordoma, extradural spinal tumors, intramedullary compression, rapidly progressive paraparesis

\section{INTRODUCTION}

Chordomas are low-grade, slow-growing, but locally invasive and aggressive malignant tumors, arising from the remnants of the embryonic notochord (1). Given its origin, the distribution of chordomas is along the axial skeleton as follows: $50 \%$ of cases in the sacral region, 35\% skull base and $15 \%$ occur in the vertebral bodies of the spine, which most commonly involve cervical (particu- larly $\mathrm{C} 2$ ), followed by lumbar, and then the thoracic spine $(2,3)$. They often extend across the intervertebral disc space, involving more than one vertebral segment and also into the epidural space compressing the spinal cord, or along the nerve roots enlarging the neural foramen.

Without a prompt and aggressive treatment, the chances of metastasis are low, but the local recurrence rate is high (about 50\%) with a $40 \%$ chance of 10-year survival. 
Our purpose is to present a rare case of an extradural thoracic chordoma, the importance of differential diagnosis and quick surgical decision as main treatment option of this case, for a better outcome.

\section{CASE PRESENTATION}

A 61-years-old male patient presented with insidious onset, non-traumatic back pain for 1-month followed by rapid progressive distal paresthesia and paraparesis for a week and $24 \mathrm{~h}$ urinary retention. He has a history of hypertension and type II diabetes mellitus, but there was no history of trauma, anorexia, fever or contact TB patients, although his back pain was first considered to be due to a recent lung infection that was treated with antibiotics

On clinical examination, he had decreased motor strength with distal asymmetric paraparesis $3 / 5$

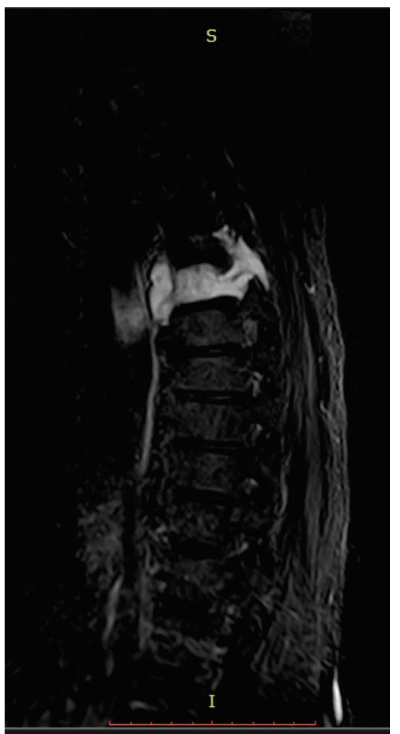

a
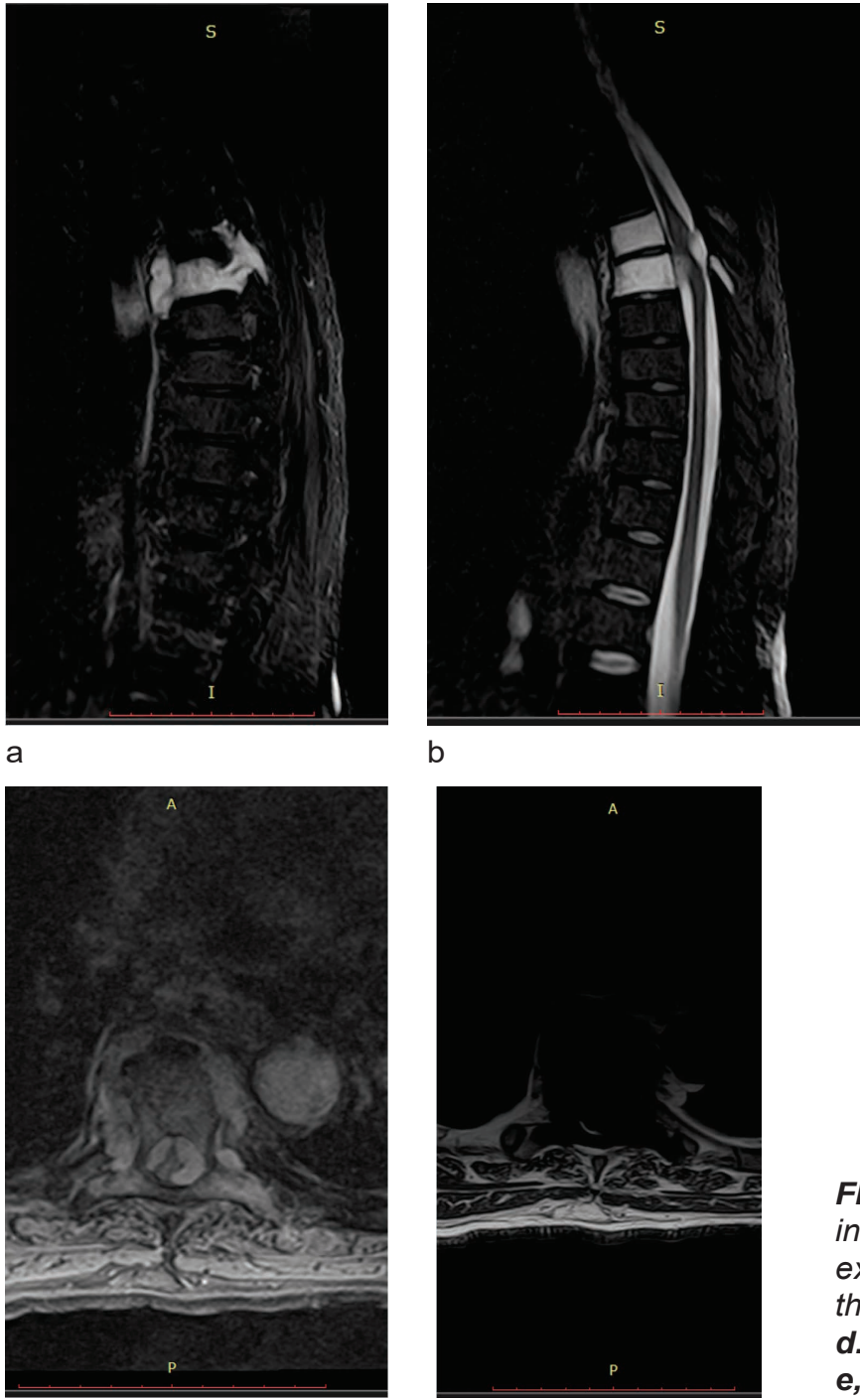

e

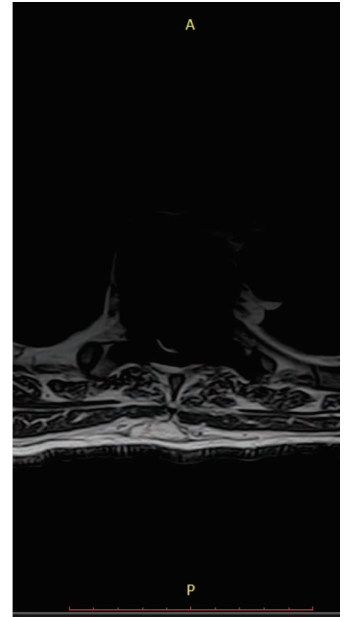

f

(Medical Research Council grade) on the left lower limb and 4/5 on the right lower limb, wich rapidly progressed to paraplegia within 24 hours, hypoesthesia with a T5-T6 level of sensibility, with diminished deep tendon reflexes in both lower limbs, no Babinski sign and no signs of muscles atrophy, ileus paraliticus and acute urine retention. Sensory examination was abnormal with inguinal and lower limbs hypoesthesia, but without mioarthrokinetic loss of sensitivity.

\section{Investigations}

Routine blood tests showed high levels of serum glucose due to his diabetes, leukocytosis, inflammatory syndrome.

The chest $X$ ray revealed bilateral infrahilar alveolitis.

The abdominal ultrasonography showed abdominal bloating, and half-empty bladder with

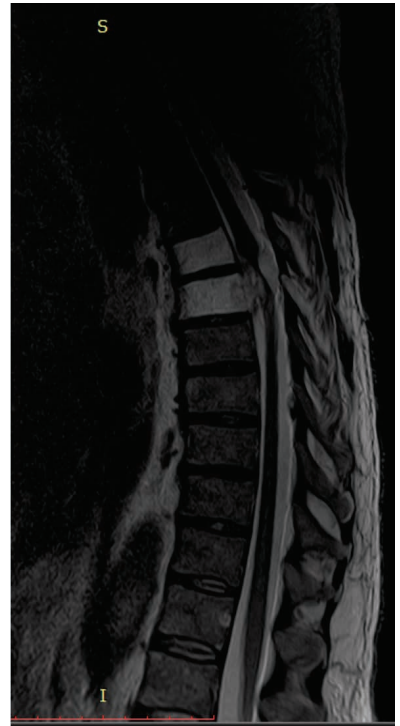

C

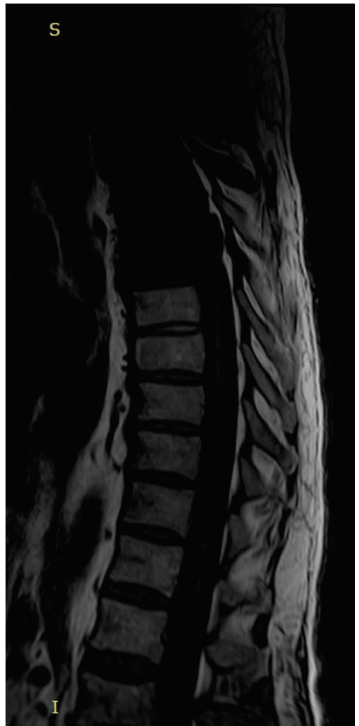

d
FIGURE 1. a,b,c. Marked T2/ STIR hyper signal that interests the vertebral bodies, the posterior arches with extension in the pre and paravertebral space and through the conjugated foramen in the epidural space d. difuse infiltration of bone marrow in T1 e,f. $51 \times 32 \times 14 \mathrm{~mm}$ contrast-enhancing lesion arising from T5 to T7 with medullar compression and myelopathy 
normal kidneys and prostate. We could not performe a urodynamic study. The head and thoracic spine CT were normal.

Due to the clinical examination that was suggestive for a lower motor neuron injury, a thoracic

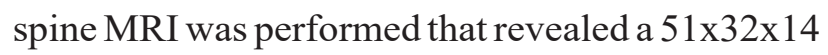
$\mathrm{mm}$ contrast-enhancing lesion arising from $\mathrm{T} 5$ to T6 vertebral level, with paravertebral extension and medullary compression (fig. 1).

After emergency surgery, the histopathological examination of a postoperative specimen indicated chordoma.

A contrast whole body CT was done to look for metastases, that came back negative.

\section{Differential diagnosis}

As differential diagnosis, we kept in mind metastatic vertebral involvement of lung cancer, breast carcinoma, thyroid carcinoma, renal cell carcinoma, osteosarcoma, chondrosarcoma, haemangioblastoma and a remote possibility of tuberculoma.

\section{Treatment}

He was transferred to the neurosurgery department, were underwent urgent decompression laminectomy for spinal cord compression, following which his neurological symptoms slightly improved within 5 days.

\section{Outcome and follow up}

In the immediate postoperative period, there was a significant reduction in pain and muscle strenght improved to $2 / 5$ (MRC) in both lower limbs. However, there was no evidence of any improvement in urinary incontinence. Patient was then referred to oncology department for further investigations, treatment and follow up.

\section{DISCUSSION}

Chordomas are primary bone tumors, which arise from notochord remnants. They can affect any part of the axial skeleton, with $35 \%$ cases occurring intracranial, $50 \%$ in the sacrum, and $15 \%$ affecting the rest of the spine (1).

They are more frequent in male (2:1) and may occur at any age, but peak incidence is in fifth or sixth decades of life (1), with an exception: the skull base presentation affects teenagers and children.
Metastasis can appear in $40 \%$ of all cases, with lungs, liver, lymph nodes and cerebrospinal (CSF) as the commonly reported sites (1). Survival is modest ranging from $50 \%$ to $68 \%$ at 5 years and $28 \%$ to $40 \%$ at 10 years, due to the local aggressive course (2).

Histologically, chordomas consist of fibrous tissue separating chords of tumor from mucoid matrix. Microscopically they are characterized by foamy cells with vacuoles. There are 3 histological subtypes: classical, chondroid and de-differentiated, the last one being the most aggressive and affecting mostly children. Immuno-histological markers are needed to establish diagnosis, for molecular target therapy and to differentiate it from other tumors like chondrosarcoma. Chordoma stains positive for nuclear brachyury along with S-100, epithelial membrane antigen, vimentin, and cytokeratin (3). They are unusual primary vertebral malignancies that must be considered among the differential diagnoses for extradural spinal tumors (4).

Chordoma is a slow growing tumor, locally invasive and destructive, that can eventually lead to compression of surrounding structures including vessels and nerves. Symptoms depend on the site involved and size of the tumor: chordomas of the skull base may present cranial nerve paresis or brain stem compression syndrome, while the other types may be suggested by the clinical signs of spinal cord compression (5).

The diagnosis workup includes radiological imaging: X-ray that can show bone erosions with irregular calcium foci along with a lytic lesion, followed by Computed tomography scan used for evaluation of the lytic lesions and locally destructive pattern with hyper dense soft tissue mass with calcification and most important MRI which is considered superior to other imaging modalities and lesions appear hypointense on $\mathrm{T} 1$, hyperintense on T2 and Flair images and may have heterogeneous contrast enhancement (6). Pathological examination is the gold standard for diagnosis of disease.

Regarding treatment options, complete surgical resection with negative margins is the gold standard and may be combined with radiotherapy (7). This leads to better disease control and reduced local recurrence (8). An incomplete surgical resection, skull base location, advanced age, and large 
size and tumor necrosis are associated with poor prognosis (9).

Radiotherapy with target dose of greater than $60 \mathrm{~Gy}$ is the option in cases where resection is not suitable. Proton beam therapy is preferred over photon therapy, due to its straighter trajectory and so minimizing damage to surrounding critical structures (10).

Chemotherapy did not show to be effective and it is not recommended, hence the focus has shifted towards investigating molecular targeted therapy as a treatment modality (11). There have been done studies with promising results on Imatinib, a platelet-derived growth factor receptor (PDGFR) TKI,
Lapatinib in EGFR-positive chordomas and Sorafenib-kinase inhibitor.

\section{CONCLUSIONS}

Chordomas must be kept in mind as differential diagnoses for extradural spinal tumors. Due to the rarity of chordomas, limited trials have been carried out and as a result, there is lack of standardized systemic and effective therapy. At present, unblock resection with possible radiotherapy remains the only effective treatment option, but still the recurrence rates are quite high and the long term prognosis is poor.

Conflict of interest: none declared Financial support: none declared

\section{REFERENCES}

1. Fletcher CDM, Bridge JA, Hogendoorn PCW, Mertens F. WHO Classification of Tumours of Soft Tissue and Bone. WHO Classification of Tumours, 4th Edition, Volume 5. Edited by IARC, 2013.

2. Sciubba DM, Chi JH, Rhines LD, Gokaslan ZL. Chordoma of the spinal column. Neurosurg Clin N Am. 2008 Jan;19(1):5-15.

3. Crapanzano JP, Ali SZ, Ginsberg MS, Zakowski MF. Chordoma: a cytologic study with histologic and radiologic correlation. Cancer. 2001 Feb 25;93(1):40-51.

4. Awuor V, Stewart CE, Camma A, Renner J, Tongson JM. Rare case of an extraosseous cervical chordoma with both intradural and extensive extraspinal involvement. Surg Neurol Int. 2017 Oct 13;8:250.

5. Walcott BP, Nahed BV, Mohyeldin A, Coumans JV, Kahle KT, Ferreira MJ. Chordoma: current concepts, management, and future directions. Lancet Oncol. 2012 Feb;13(2):e69-76.

6. Khawaja AM, Venkatraman A, Mirza M. Clival Chordoma: Case Report and Review of Recent Developments in Surgical and Adjuvant Treatments. Pol J Radiol. 2017 Nov 17;82:670-675.
7. Jawad MU, Scully SP. Surgery significantly improves survival in patients with chordoma. Spine (Phila Pa 1976). 2010 Jan 1;35(1):117-23.

8. Alan O, Akin Telli T, Ercelep O, Tanrikulu Simsek E, Basoglu Tuylu T, Mutis A, Hasanov R, Kaya S, Akgül Babacan N, Dane F, Yumuk PF. Chordoma: a case series and review of the literature. J Med Case Rep. 2018 Aug 27;12(1):239.

9. Gay E, Sekhar LN, Rubinstein E, Wright DC, Sen C, Janecka IP, Snyderman $\mathrm{CH}$. Chordomas and chondrosarcomas of the cranial base: results and follow-up of 60 patients. Neurosurgery. 1995 May;36(5):887-97.

10. Frank SJ, Selek U. Proton beam radiation therapy for head and neck malignancies. Curr Oncol Rep. 2010 May;12(3):202-7.

11. Fleming GF, Heimann PS, Stephens JK, Simon MA, Ferguson MK, aggressive chemotherapy in two cases. Cancer. 1993 Aug 1;72(3):714-8. Benjamin RS, Samuels BL. Dedifferentiated chordoma. Response to 